\title{
Modelling scenarios of the epidemic of COVID-19 in Canada
}

\author{
Nick H Ogden ${ }^{1 \star}$, Aamir Fazil', Julien Arino², Philippe Berthiaume ${ }^{1}$, David N Fisman ${ }^{3}$, Amy L Greer ${ }^{4}$, \\ Antoinette Ludwig', Victoria $\mathrm{Ng}^{1}$, Ashleigh R Tuite ${ }^{3}$, Patricia Turgeon', Lisa A Waddell', \\ Jianhong $\mathrm{Wu}^{5,6}$
}

\begin{abstract}
Background: Severe acute respiratory syndrome virus 2 (SARS-CoV-2), likely a bat-origin coronavirus, spilled over from wildlife to humans in China in late 2019, manifesting as a respiratory disease. Coronavirus disease 2019 (COVID-19) spread initially within China and then globally, resulting in a pandemic.

Objective: This article describes predictive modelling of COVID-19 in general, and efforts within the Public Health Agency of Canada to model the effects of non-pharmaceutical interventions (NPIs) on transmission of SARS-CoV-2 in the Canadian population to support public health decisions.
\end{abstract}

Methods: The broad objectives of two modelling approaches, 1) an agent-based model and 2) a deterministic compartmental model, are described and a synopsis of studies is illustrated using a model developed in Analytica 5.3 software.

Results: Without intervention, more than $70 \%$ of the Canadian population may become infected. Non-pharmaceutical interventions, applied with an intensity insufficient to cause the epidemic to die out, reduce the attack rate to $50 \%$ or less, and the epidemic is longer with a lower peak. If NPIs are lifted early, the epidemic may rebound, resulting in high percentages (more than $70 \%$ ) of the population affected. If NPIs are applied with intensity high enough to cause the epidemic to die out, the attack rate can be reduced to between $1 \%$ and $25 \%$ of the population.

Conclusion: Applying NPIs with intensity high enough to cause the epidemic to die out would seem to be the preferred choice. Lifting disruptive NPIs such as shut-downs must be accompanied by enhancements to other NPIs to prevent new introductions and to identify and control any new transmission chains.
This work is licensed under a Creative Commons Attribution 4.0 International License.

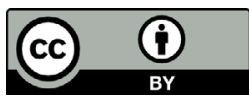

Affiliations

${ }^{1}$ Public Health Risk Sciences Division, National Microbiology Laboratory, Public Health Agency of Canada, St. Hyacinthe, QC and Guelph, ON

${ }^{2}$ Department of Mathematics \& Data Science NEXUS, University of Manitoba, Winnipeg, MB

${ }^{3}$ Dalla Lana School of Public Health, University of Toronto, Toronto, ON

${ }^{4}$ Department of Population Medicine, University of Guelph, Guelph, ON

${ }^{5}$ Laboratory for Industrial and Applied Mathematics, Department of Mathematics and Statistics, York University, Toronto, ON

${ }^{6}$ Fields-CQAM Laboratory of Mathematics for Public Health, York University, Toronto, ON

${ }^{\star}$ Correspondence:

nicholas.ogden@canada.ca

Suggested citation: Ogden NH, Fazil A, Arino J, Berthiaume P, Fisman DN, Greer AL, Ludwig A, Ng V, Tuite AR, Turgeon P, Waddell LA, Wu J. Modelling scenarios of the epidemic of COVID-19 in Canada. Can Commun Dis Rep 2020;46(6):198-204. https://doi.org/10.14745/ccdr.v46i06a08

Keywords: modelling, COVID-19, Canada, non-pharmaceutical interventions

\section{Introduction}

In this article, we review efforts within the Public Health Agency of Canada (PHAC) to model transmission of severe acute respiratory syndrome virus 2 (SARS-CoV-2), the agent of coronavirus disease 2019 (COVID-19), to support public health decisions. The COVID-19 pandemic has emerged at remarkable speed. The SARS-CoV-2 is likely a bat-origin coronavirus (1) that may have "spilled over" into humans from an intermediary animal reservoir not yet identified. The first detected human exposure event was linked to a "wet market" in the city of Wuhan, the capital city of the province of Hubei, China some time during late 2019 (2). The virus was likely already capable of human-to-human transmission, but evolved more efficient transmissibility during late 2019 (3). Human-to-human transmission was officially recognised by the global public health community in mid-January 2020 (4). Shortly after this, spatial spread in China was reported. Intense public health measures (or non-pharmaceutical interventions; NPIs) of case detection, contact tracing and quarantine, and 
social distancing were implemented within the province of Hubei, and the region was isolated from the rest of China by travel restrictions (5). International travel restrictions to and from China were introduced but cases had already escaped outside of China (6), and the consequent global spread resulted in declaration of a pandemic (4). The first travel-related cases were identified in Canada in January 2020 and by April 2020, community transmission was occurring in all provinces with the possible exception of Prince Edward Island. Community transmission has yet to be reported from the territories. The majority of cases and deaths have been reported from the four largest provinces (British Columbia, Alberta, Ontario and Quebec). Physical distancing (including school, college, university and "non-essential" business closures) was implemented from mid-March 2020 in Canada and subsequent reductions in disease transmission suggest that these and other NPIs (detailed below) are slowing the epidemic (7).

\section{Evolution of COVID-19 modelling}

At first, modelling studies focused on the epidemic in China, and particularly on the dynamics of the epidemic in the city of Wuhan and throughout the province of Hubei. At this early stage, there was much effort to analyse surveillance data from China to obtain parameter estimates such as the basic reproduction number $\left(R_{0}\right)$, case fatality rate and incubation period (8). For the first attempts at Susceptible-Exposed-Infectious-Recovered (SEIR) type dynamic models, parameter estimates were "borrowed" from what was known about other coronaviruses (SARS-CoV and MERS-CoV) (6) and/or obtained by fitting the models to surveillance data $(9,10)$. As more data on SARS-CoV-2 virus transmission and the course of infection in humans have become available, models have become increasingly parameterised using SARS-CoV-2-specific data (11). With global spread of the disease, and with a vaccine likely more than a year away, modelling efforts turned to assessing the possible extent of the epidemic in countries outside China, and the impact of different NPIs $(11,12)$. Emerging science has revealed that SARS-CoV-2 virus is highly transmissible by respiratory and possibly fecaloral routes, is transmitted before symptoms appear and some cases may be entirely asymptomatic $(13,14)$. The virus can be highly pathogenic for older people and some younger people, particularly those with co-morbidities (14). Presymptomatic transmission, mild symptoms (particularly in younger age groups) and asymptomatic cases all hinder detection of infective cases (in contrast to SARS) (15), making control difficult. Modelling efforts to date have illuminated the magnitude of the challenge we face: 1$)$ the global population is entirely immunologically naive, 2 ) the virus is very highly transmissible $\left(R_{0}\right.$ values may be greater than five in some settings) (16) and 3) the level of pathogenicity of SARS-CoV-2 means even the most advanced healthcare systems in the world may be completely overwhelmed if the virus is allowed to spread without introducing NPIs. At the same time, and in contrast to pandemic influenza, there are no known effective antivirals.

\section{COVID-19 modelling in Canada}

Predictive modelling of COVID-19 by Canadian scientists is a field of study that was approximately three months old at the time of writing (early May 2020), but there is extensive skill in Canada in modelling the transmission of infectious diseases. Some previously-developed models that investigated interventions to control H1N1 and other influenzas $(17,18)$ have been adapted to assess the transmission of SARS-CoV-2 and the impacts of different NPIs $(9,19,20)$. Tang et al. (9) and Li et al. (21) have developed SEIR-type models to explore transmission and learn from NPIs implemented in China and South Korea. An Expert Modelling Group, comprising more than 50 federal, provincial, territorial and university-based modellers and epidemiologists, has been assembled by PHAC to develop a Canadian COVID-19 modelling network that supports decision-making. Similar groups have been developed in other countries, with liaison between them facilitated by a World Health Organization modelling group.

\section{COVID-19 modelling at PHAC}

In January 2020, a modelling group was convened, and development of two complementary modelling approaches was initiated. The prime objective of this modelling was to assess the impact of different NPIs and levels of efficacy needed to control the epidemic in Canada. In the absence of a vaccine or treatment, the NPIs available to control the epidemic, which were explored in modelling, are 1) physical distancing (closure of schools, colleges and universities, meeting places, gatherings and personal distancing) that reduces the rates of contact between members of society (including those who may be infected), 2) detection of cases by surveillance, and their isolation to prevent them from transmitting infection and 3) tracing and quarantine of people who have had contacts with cases.

The two modelling approaches used will be published as separate, more detailed articles, and are termed "approaches" as the models themselves have evolved with evolving knowledge. What follows is a broad description of these approaches, which are based on 1) an agent-based model (22) and 2) a deterministic compartment model (23). The agent-based approach was developed de novo using AnyLogic ${ }^{\odot}$ software, while the deterministic model runs in $\mathrm{R}(24)$. Initial versions of the deterministic model were adapted from Tang et al. (9). Both are SEIR-type models with elements to model SARS-CoV-2 and impacts of NPIs, with more realism (Figure 1). These elements include compartments for isolated cases and quarantined "exposed" contacts from which onward transmission to susceptible people is limited or absent, compartments for 
Figure 1: A schematic of the Analytica 5.3 compartment model showing the flow of populations between compartments

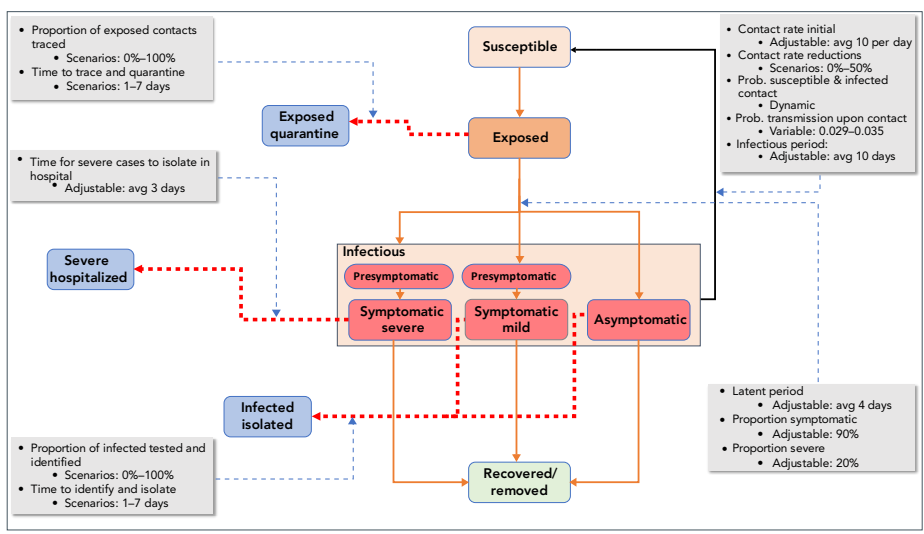

Abbreviations: avg, average; prob, probable

The black arrow reflects the transmission between infected and susceptible populations, the red dotted lines reflect the removal of infectious or exposed individuals as a result of non-pharmaceutical interventions

asymptomatic cases that may or may not be detected by surveillance, as well as flows to "isolation" and "quarantine" compartments that allow variation according to different levels of public health effort. Parameters in the models are calibrated according to values obtained by literature searches, which are conducted each day to ensure evolving knowledge is captured by the models.

In the deterministic modelling approach, effects of physical distancing are modelled in a simplistic way by reducing daily per capita contact rates informed by a number of metrics including cellphone data (25). The agent-based model approach, which contains stochastic elements, uses a more detailed representation of communities, including homes and communal meeting spaces that may represent workplaces, schools, malls and restaurants etc., so this model is capable of modelling effects of closures in more detail and predicting different realizations of disease spread in the community. The agent-based approach includes contact rates within and between age groups that are (20) based on the POLYMOD study (26). Contact data specific for the United Kingdom (UK) and European countries were used as a surrogate for contact rates in Canada given that similar studies have not been conducted in Canada. The deterministic approach has used homogenous mixing but is also being modified to include contact rates that vary within and between age groups. For illustrative purposes, a deterministic compartment model has been developed in Analytica 5.3 (Lumina Inc.) using the knowledge of COVID-19 transmission elucidated by the two modelling approaches. Code for this model is available upon request with instructions on how to explore the model and generate results.

Key outputs from the models are 1) under what circumstances the NPIs cause the epidemic to die out by reducing the effective reproduction rate $\left(R_{\mathrm{e}}\right)$ below unity (i.e. one infected person infects fewer than one other person, on average) and 2) the final attack rate (i.e. the total percentage of the population infected) and 3 ) the approximate duration of the epidemic.

\section{Synopsis of findings of modelling conducted within and outside Public Health Agency of Canada}

The following synopsis of results of modelling studies includes those conducted by PHAC with reference to studies conducted by modelling groups outside the Government of Canada. The outputs are illustrated here using graphs generated by the model developed in Analytica 5.3.

\section{What happens in the absence of NPIs?}

The baseline to compare impacts of NPIs is a scenario where there is no effort to control disease spread. In this case, the attack rate is predicted to be greater than $70 \%$, and the epidemic lasts approximately one year (Figure 2). These findings are consistent with studies estimating impacts on UK and United States populations (11).

Figure 2: Impacts of partially-effective NPIs on the epidemic compared to the baseline with no control efforts

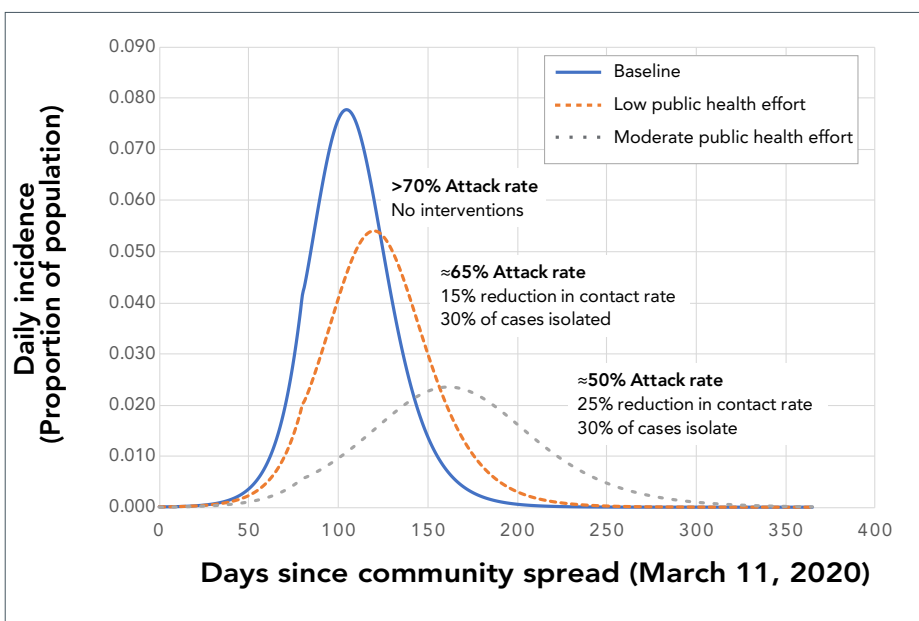

Values by each curve are the final estimated attack rate and the non-pharmaceutical intervention (NPIs) employed. It is assumed that NPIs are in effect for the duration of the epidemic

\section{What happens when NPIs are partially effective?}

If NPIs, maintained throughout the epidemic, are partially effective (i.e. they have impacts on the epidemic but do not cause it to die out), the main effects are as follows: the epidemic is prolonged, the peak is reduced, the epidemiological curve is flattened and the attack rate is reduced to approximately $50 \%$ (to $25 \%$ in some models) (20) (Figure 2). This finding is consistent with equivalent modelling studies $(11,20)$. This scenario has been termed "delay and reduce." 
If the NPIs do not cause the epidemic to die out and are lifted before the epidemic is over, the epidemic is predicted to rebound and the attack rate can be as high as without NPIs because the majority of the population remains naive (Figure 3) $(11,20)$.

Figure 3: The effect of initiating partially-effective non-pharmaceutical interventions (in this case physical distancing), and then removing these interventions before the epidemic ends ${ }^{a}$

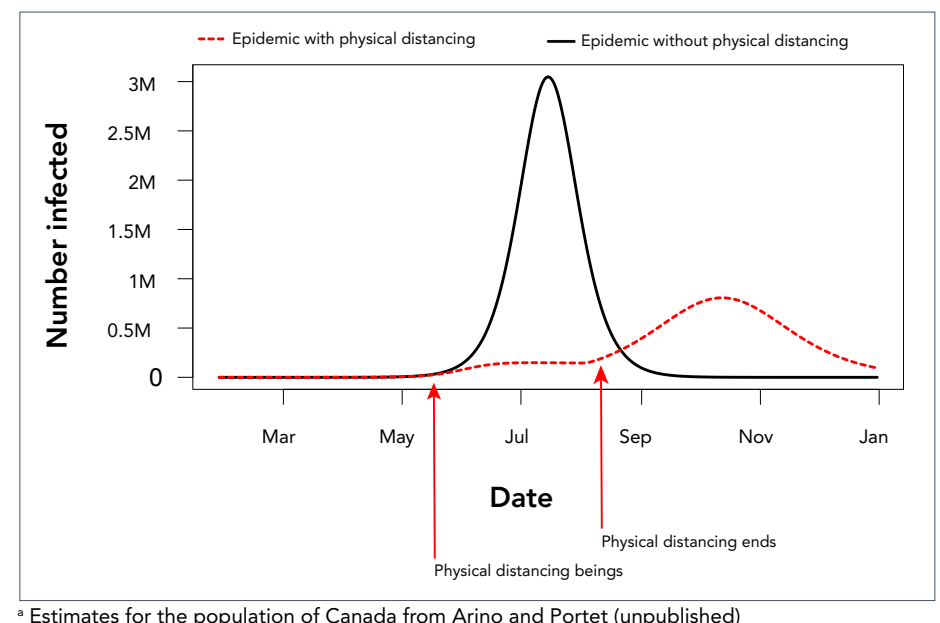

Estimates for the population of Canada from Arino and Portet (unpublished)

3. What happens when NPIs are highly effective?

When NPIs are highly effective, $R_{e}$ falls below unity, the epidemic dies out and does not rebound if NPIs are lifted (also referred to as epidemic control). How soon that happens, and thus the final attack rate (which may be anywhere between less than $1 \%$ and $25 \%$ ), depends on a number of factors including at what point in the epidemic NPIs are implemented, the intensity with which NPIs are implemented, the duration of implementation and the level of compliance (Figure 4) (12).

\section{Assessing hospitalisation and mortality rates from attack rates}

The main objective of the modelling approaches was to compare the impacts of different NPIs. There remains much uncertainty in some model parameters and their distributions, including the duration of the latent period, the proportion of cases that are asymptomatic and the duration of infectivity. The strength of these models is their ability to compare amongst different NPIs using current best estimates of parameter values. However, in order to ensure health care capacity is sufficient to respond to the pandemic, planners need to have a range of estimates for the expected numbers of cases, hospitalizations, cases needing care in intensive care units (ICU) and fatalities. Initial modelling approaches focused on estimating total attack rates, and were not designed to estimate hospitalizations, cases
Figure 4: Effects of different levels and combinations of non-pharmaceutical interventions on whether or not, and how quickly, epidemic control is reached

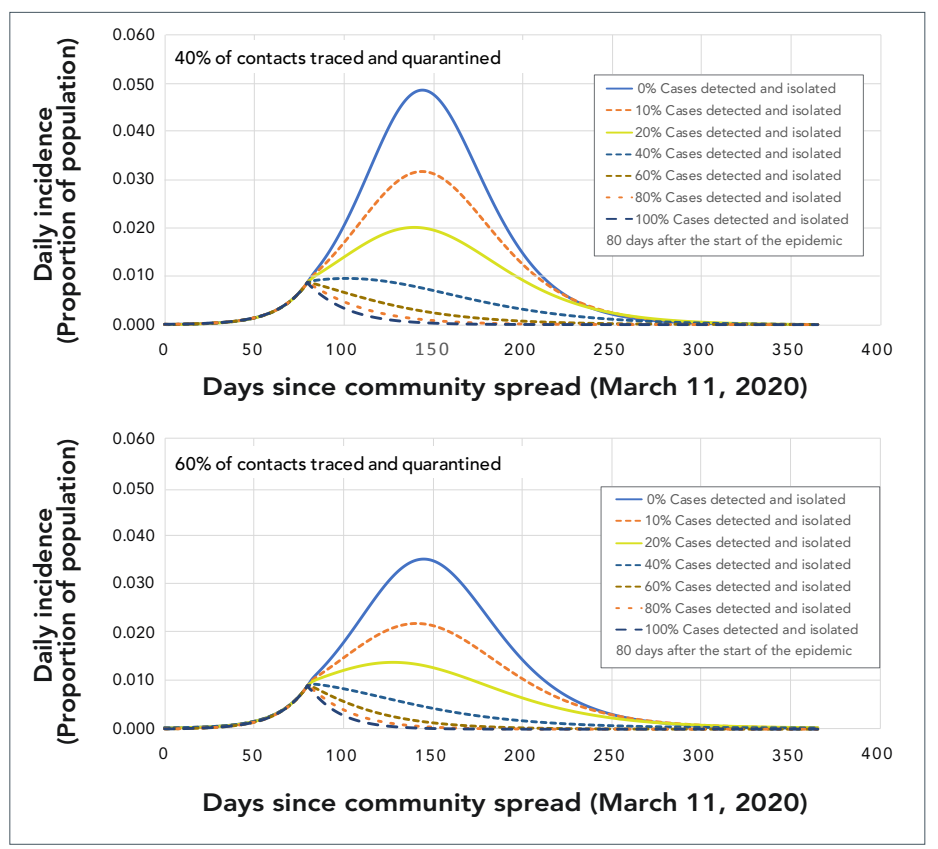

needing ICU care and fatalities. To obtain these estimates from total attack rates, age-specific severity estimates from analysis of international surveillance data (27) were used to assess the proportion of cases in Canada that would be mild or asymptomatic, require hospitalization or ICU treatment, and may die, according to the demography of the Canadian population as a whole (28). The estimates per one million population are shown in Table 1.

These estimates are crude and more precise health care needs estimates should be calculated at the community level (e.g. catchment area for a hospital or group of hospitals) so the specific age structure and co-morbidities of the community under evaluation can be accounted for in the model (19).

\section{Observations from Canada and elsewhere in the world}

The outputs of modelling studies are theoretical, but their insights and policy implications have been bolstered by realworld evidence. Epidemic control has been realised in Singapore, China and South Korea, with $R_{\mathrm{e}}$ falling below unity by application of a prompt and intense level of NPIs $(5,21,29)$. In contrast, in Europe, NPIs to date do not seem to have brought $R_{e}$ below unity (30). At the time of writing, in Canada the epidemic is geographically heterogeneous, but unpublished estimates suggest that in some jurisdictions $R_{\mathrm{e}}$ may be below unity, while in others this state has not been reached (unpublished; Dr. Ashleigh Tuite and Dr. David Champredon). At the time of writing, the observed case fatality rate is higher than that predicted using 
Table 1: Estimates per million population of hospitalisations, patients needing intensive care unit care and fatalities according to different scenarios for levels of control ${ }^{a}$

\begin{tabular}{|c|c|c|c|c|c|c|}
\hline Level of epidemic control & \multicolumn{3}{|c|}{ "Delay and reduce" } & \multicolumn{3}{|c|}{ Epidemic controlled } \\
\hline Attack rate & $50 \%$ & $25 \%$ & $10 \%$ & $5 \%$ & $2.5 \%$ & $1 \%$ \\
\hline All cases & 500,000 & 250,000 & 100,000 & 50,000 & 25,000 & 10,000 \\
\hline Mild (89.5\%) & 450,000 & 225,000 & 90,000 & 45,000 & 22,000 & 9,000 \\
\hline Hospitalised-not ICU (8\%) & 39,000 & 19,000 & 7,800 & 3,900 & 2,000 & 800 \\
\hline Hospitalised-ICU (2.5\%) & 12,000 & 6,000 & 2,400 & 1,200 & 600 & 200 \\
\hline Fatalities ( $1.2 \%$ all cases) & 6,000 & 3,000 & 1,200 & 600 & 300 & 100 \\
\hline
\end{tabular}

Abbreviation: ICU, intensive care unit

a Figures greater than 1,000 were rounded to the nearest 1,000

methods described above because of extensive transmission in long-term care and seniors facilities. In these facilities, contact rates are likely very high (31) and the population very vulnerable to COVID-19.

\section{Conclusion}

The modelling studies described here provide information for planning of public health policies to combat the unprecedented risk of COVID-19 to the health and well-being of Canadians and the rest of the world. These studies underline that without NPIs, the majority of Canadians would acquire infection in a relatively short period of time, and the health care system would most likely be overwhelmed, resulting in a higher case fatality rate, particularly in the most vulnerable age groups. The intensity of the NPIs, and the compliance of the public, will determine whether the epidemic is brought under control, or delayed and reduced. The former would seem the preferred objective as the numbers of Canadians affected would be minimized. However, this will require a very high degree of public health effort and public buy-in and, if successful, will require a high level of vigilance to identify imported cases and any transmission chains that may result, because the Canadian population remains largely infection naive. If transmission in Canada is not completely extinguished, strong NPIs will have to remain in place or the epidemic will rebound. Any lifting of physical distancing, which appears to be bearing fruit at present, will have to be matched by increased efforts to detect cases by surveillance and to trace and quarantine contacts.

Modelling studies are not predictions, they present plausible outcomes with different levels of NPIs, given our current knowledge of the virus and its transmission, and can be used to support planning, particularly in evidence-limited situations, such as emerging infectious disease epidemics. Our knowledge is constantly evolving, and the models and their outcomes will evolve accordingly. The models provide information that is useful for decision-making, but they do not make decisions. Decisions on public health programs to control COVID-19 in Canada will be made accounting for a range of additional factors that include (but are not limited to) economic impacts, ethical and legal concerns, and the negative health impacts of aggressive physical distancing.

\section{Funding}

This work was funded by the Public Health Agency of Canada. JA and JW's work has also been funded by the Canadian Institute of Health Research (CIHR) 2019 Novel Coronavirus (COVID-19) rapid research program.

\section{References}

1. Lau SK, Luk HK, Wong AC, Li KS, Zhu L, He Z, Fung J, Chan TT, Fung KS, Woo PC. Possible Bat Origin of Severe Acute Respiratory Syndrome Coronavirus 2. Emerg Infect Dis 2020;26(7). DOI [Epub ahead of print] PubMed

2. Zhang T, Wu Q, Zhang Z. Probable Pangolin Origin of SARS-CoV-2 Associated with the COVID-19 Outbreak. Curr Biol 2020 Apr;30(7):1346-1351.e2. DOI PubMed

3. Andersen KG, Rambaut A, Lipkin WI, Holmes EC, Garry RF. The proximal origin of SARS-CoV-2. Nat Med 2020;26(4):450-2. DOI PubMed

4. World Health Organization. WHO Timeline - COVID-19. Geneva (CH): WHO; April 27, 2020. https://www.who.int/ news-room/detail/08-04-2020-who-timeline---covid-19

5. Wang C, Liu L, Hao X, Guo H, Wang Q, Huang J, He N, Yu H, Lin X, Pan A, Wei S, Wu T. Association of Public Health Interventions With the Epidemiology of the COVID-19 Outbreak in Wuhan, China. JAMA 2020;323(19):1915-23. $\mathrm{DOI}$

6. Wu JT, Leung K, Leung GM. Nowcasting and forecasting the potential domestic and international spread of the 2019-nCoV outbreak originating in Wuhan, China: a modelling study. Lancet 2020;395(10225):689-97. DOI PubMed

7. Government of Canada. Coronavirus disease (COVID-19). Ottawa (ON); Government of Canada; modified May 15, 2020. https://www.canada.ca/en/public-health/services/ diseases/coronavirus-disease-covid-19.html 
8. Li J, Wang Y, Gilmour S, Wang M, Yoneoka D, Wang Y, You X, Gu J, Hao C, Peng L, Du Z, Xu DR, Hao Y. Estimation of the epidemic properties of the 2019 novel coronavirus: $A$ mathematical modeling study. MedRXiv: 2020a https://www. medrxiv.org/content/10.1101/2020.02.18.20024315v1.full. pdf

9. Tang B, Wang X, Li Q, Bragazzi NL, Tang S, Xiao Y, Wu J. Estimation of the Transmission Risk of the 2019-nCoV and Its Implication for Public Health Interventions. J Clin Med 2020a;9(2):E462. DOl PubMed

10. Kucharski AJ, Russell TW, Diamond C, Liu Y, Edmunds J, Funk S, Eggo RM; Centre for Mathematical Modelling of Infectious Diseases COVID-19 Working Group. Early dynamics of transmission and control of COVID-19: a mathematical modelling study. Lancet Infect Dis. 2020;20(5)552-8. DOI [Epub ahead of print]

11. Ferguson NM, Laydon D, Nedjati-Gilani G, Imai N, Ainslie K, Baguelin M, Bhatia S, Boonyasiri A, Cucunubá Z, Cuomo-Dannenburg G, Dighe A, Dorigatti I, Fu H, Gaythorpe K, Green W, Hamlet A, Hinsley W, Okell LC, van Elsland S, Thompson H, Verity R, Volz E, Wang H, Wang Y, Walker PG, Walters C, Winskill P, Whittaker C, Donnelly CA, Riley S, Ghani AC. Impact of non-pharmaceutical interventions (NPIs) to reduce COVID-19 mortality and healthcare demand. London (UK): Imperial College; 2020. https://www.imperial.ac.uk/media/imperial-college/ medicine/sph/ide/gida-fellowships/Imperial-CollegeCOVID19-NPI-modelling-16-03-2020.pdf

12. Hellewell J, Abbott $\mathrm{S}$, Gimma $\mathrm{A}$, Bosse $\mathrm{NI}$, Jarvis $\mathrm{Cl}$, Russell TW, Munday JD, Kucharski AJ, Edmunds WJ, Funk S, Eggo RM; Centre for the Mathematical Modelling of Infectious Diseases COVID-19 Working Group. Feasibility of controlling COVID-19 outbreaks by isolation of cases and contacts. Lancet Glob Health 2020;8(4):e488-96.

DOI PubMed

13. Wei WE, Li Z, Chiew CJ, Yong SE, Toh MP, Lee VJ. Presymptomatic Transmission of SARS-CoV-2 - Singapore, January 23-March 16, 2020. MMWR Morb Mortal Wkly Rep 2020;69(14):411-5. DOl PubMed

14. Vetter P, Vu DL, L'Huillier AG, Schibler M, Kaiser L, Jacquerioz F. Clinical features of covid-19. BMJ 2020;369:m1470. DOl PubMed

15. Wilder-Smith A, Chiew CJ, Lee VJ. Can we contain the COVID-19 outbreak with the same measures as for SARS? Lancet Infect Dis 2020 May;20(5):e102-7. DOI PubMed

16. Sanche S, Lin YT, Xu C, Romero-Severson E, Hengartner N, Ke R. High Contagiousness and Rapid Spread of Severe Acute Respiratory Syndrome Coronavirus 2. Emerg Infect Dis 2020;26(7). DOI [Epub ahead of print] PubMed

17. Arino J, Brauer F, van den Driessche P, Watmough J, Wu J. A model for influenza with vaccination and antiviral treatment. J Theor Biol 2008;253(1):118-30. DOl PubMed
18. Tuite A, Fisman DN, Kwong JC, Greer A. Optimal pandemic influenza vaccine allocation strategies for the canadian population. PLoS Curr 2010;2:RRN1144. DOI PubMed

19. Arino J, Portet S. A simple model for COVID-19. Inf Dis Modelling. 2020:5: 309-15. PubMed

20. Tuite AR, Fisman DN, Greer AL. Mathematical modelling of COVID-19 transmission and mitigation strategies in the population of Ontario, Canada. CMAJ 2020;192(19):E497505. DOI PubMed

21. Li J, Yuan P, Heffernan J, Zheng T, Ogden N, Sander B, Li J, Li Q, Bélair J, Dzevela Kong J, Aruffo E, Tan Y, Jin Z, Yu Y, Fan M, Cui J, Teng Z, Zhu H. Observation wards and control of the transmission of COVID-19 in Wuhan. Bull World Health Organ. DOI

22. Ng V, Fazil A, Waddell S, Bancej C, Turgeon P, Ogden NH. An agent-based model of COVID-19 transmission in Canada: forecasting impacts and de-escalation of non-pharmaceutical public health interventions [submitted for publication].

23. Ludwig $A$, Berthiaume $P$, Orpana $H$, Swerdfeger $H$, Otten A, Statistics Canada team, Ogden NH. A dynamic compartmental model of national COVID-19 transmission and the impact of varying levels of case identification and contact tracing in Canada [submitted for publication].

24. R Core Team. R: A language and environment for statistical computing. Vienna (Austria): R Foundation for Statistical Computing; 2020. https://www.r-project.org/

25. Google. https://www.google.com/covid19/mobility/

26. Mossong J, Hens $N$, Jit $M$, Beutels $P$, Auranen $K$, Mikolajczyk R, Massari M, Salmaso S, Tomba GS, Wallinga J, Heijne J, Sadkowska-Todys M, Rosinska M, Edmunds WJ. Social contacts and mixing patterns relevant to the spread of infectious diseases. PLoS Med 2008;5(3):e74. DOI PubMed

27. Verity R, Okell LC, Dorigatti I, Winskill P, Whittaker C, Imai N, Cuomo-Dannenburg G, Thompson $\mathrm{H}$, Walker PGT, Fu H, Dighe A, Griffin JT, Baguelin M, Bhatia S, Boonyasiri A, Cori A, Cucunubá Z, FitzJohn R, Gaythorpe K, Green W, Hamlet A, Hinsley W, Laydon D, Nedjati-Gilani G, Riley S, van Elsland S, Volz E, Wang H, Wang Y, Xi X, Donnelly CA, Ghani AC, Ferguson NM. Estimates of the severity of coronavirus disease 2019: a model-based analysis. Lancet Infect Dis 2020:pii:S1473-3099(20)30243-7. DOI [Epub ahead of print]

28. Statistics Canada. Population estimates on July 1st, by age and sex. Table 17-10-0005-01. Ottawa (ON): StatsCan; modified May 18, 2020. https://www150.statcan.gc.ca/t1/ tbl1/en/tv.action?pid=1710000501

29. Tang B, Xia F, Bragazzi NL, McCarthy Z, Wang X, He S, Sun X, Tang S, Xiao Y, Wu J. Lessons drawn from China and South Korea for managing COVID-19 epidemic: insights from a comparative modeling study. Bull World Health Organ 2020. DOI [Epub ahead of print] 
30. Flaxman S, Mishra S, Gandy A, Unwin HJ, Coupland H, Mellan TA, Zhu H, Berah T, Eaton JW, Guzman PN, Schmit N, Callizo L, Ainslie KE, Baguelin M, Blake I, Boonyasiri A, Boyd O, Cattarino L, Ciavarella C, Cooper L, Cucunubá Z, Cuomo-Dannenburg G, Dighe A, Djaafara B, Dorigatti I, van Elsland S, FitzJohn R, Fu H, Gaythorpe K, Geidelberg L, Grassly N, Green W, Hallett T, Hamlet A, Hinsley W, Jeffrey B, Jorgensen D, Knock E, Laydon D, Nedjati-Gilani G, Nouvellet P, Parag K, Siveroni I, Thompson H, Verity R, Volz E, Walters $C$, Wang $H$, Wang $Y$, Watson $O$, Winskill $P, X i X$, Whittaker C, Walker PG, Ghani A, Donnelly CA, Riley S, Okell LC, Vollmer MA, Ferguson NM, Bhatt S. Report 13: Estimating the number of infections and the impact of non-pharmaceutical interventions on COVID-19 in 11 European countries. London (UK): Imperial College; 2020. https://www.imperial.ac.uk/mrc-global-infectious-diseaseanalysis/covid-19/report-13-europe-npi-impact/
31. Najafi M, Laskowski M, de Boer PT, Williams E, Chit A, Moghadas SM. The effect of individual movements and interventions on the spread of influenza in long-term care facilities. Med Decis Making 2017;37(8):871-81. DOI PubMed

\section{PREVENT THE SPREAD OF COVID-19}

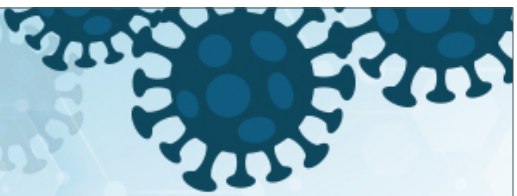

CORONAVIRUS DISEASE (COVID-19): HOW TO CARE FOR A PERSON WITH COVID-19 AT HOMEADVICE FOR CAREGIVERS

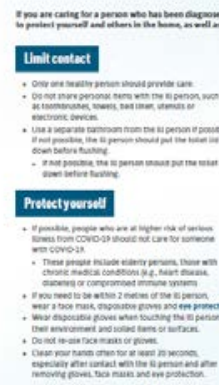

$191=$

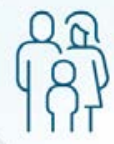

ABOUT CORONAVIRUS DISEASE (COVID-19)

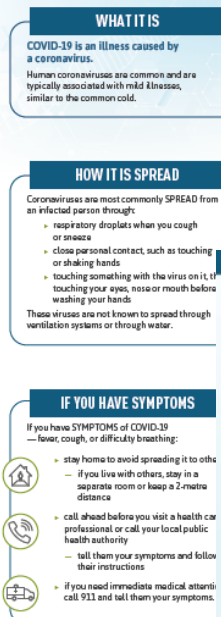

FOR MORE INFORI 1.833.784.4397

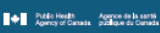
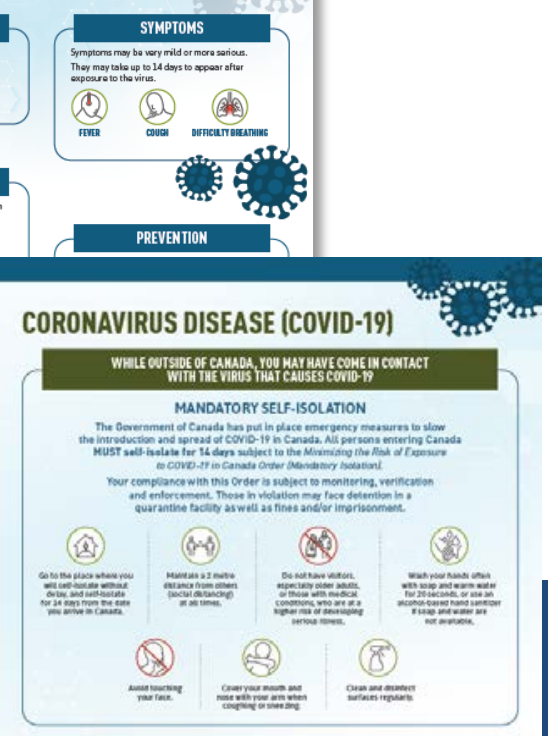

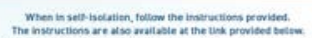

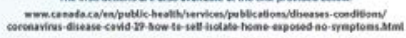
MOWITOR YOUR MELUTH FOR HOATS

\section{COVID-19}

\section{Awareness Resources}

- Get the latest guidance and awareness resources

- Some resources are available in multiple languages

\section{Visit} www.canada.ca/coronavirus 